\title{
Photonic crystal fibers: fundamental properties and applications within sensors
}

Jensen, Jesper Bo Damm; Riishede, Jesper; Broeng, Jes; Lægsgaard, Jesper; Alkeskjold, Thomas Tanggaard; Sørensen, Niels Thorkild; Hougaard, Kristian G.; Knudsen, Erik; Libori, Stig Eigil Barkou; Bjarklev, Anders Overgaard

Published in:

IEEE Sensors

Link to article, DOI:

10.1109/ICSENS.2003.1278941

Publication date:

2003

Document Version

Publisher's PDF, also known as Version of record

Link back to DTU Orbit

Citation (APA):

Jensen, J. B. D., Riishede, J., Broeng, J., Lægsgaard, J., Alkeskjold, T. T., Sørensen, N. T., Hougaard, K. G., Knudsen, E., Libori, S. E. B., \& Bjarklev, A. O. (2003). Photonic crystal fibers: fundamental properties and applications within sensors. In IEEE Sensors (Vol. 1, pp. ID1562). IEEE. https://doi.org/10.1109/ICSENS.2003.1278941

\section{General rights}

Copyright and moral rights for the publications made accessible in the public portal are retained by the authors and/or other copyright owners and it is a condition of accessing publications that users recognise and abide by the legal requirements associated with these rights.

- Users may download and print one copy of any publication from the public portal for the purpose of private study or research.

- You may not further distribute the material or use it for any profit-making activity or commercial gain

- You may freely distribute the URL identifying the publication in the public portal 


\title{
Photonic crystal fibers; fundamental properties and applications within sensors.
}

\author{
J.B. Jensen $^{+}$, J. Riishede $^{+}$, J. Broeng ${ }^{\times}$, J. Laegsgaard $^{+}$, T. Tanggaard Larsen ${ }^{+}$, \\ T. Sørensen ${ }^{+}$, K. Hougaard ${ }^{+}$, E. Knudsen ${ }^{+}$, S.B. Libori ${ }^{+}$, and A. Bjarklev ${ }^{+}$ \\ ${ }^{+}$) Research Center COM, DTU Building 345v, Kgs. Lyngby, Denmark, jbj@com.dtu.dk \\ ${ }^{\times}$) Crystal Fibre A/S, Blokken 84, DK-3460 Birkerød, Denmark
}

\section{Summary}

Since the first experimental demonstration of a Photonic Crystal Fiber (PCF) in 1996 by Knight et al. ${ }^{1}$, the optical properties and the fabrication of such fibers have attracted significant attention. The fiber structure with a lattice of air holes running along the length of the fiber provides a large variety of novel optical properties and improvements compared to standard optical fibers. The stack-and-pull procedure used to manufacture PCFs is a highly flexible method offering a large degree of freedom in the fabrication of PCFs with specific characteristics. A few of the remarkable optical properties of silica based PCFs are described and their applications within sensors are summarized.

\section{Fabrication of Photonic Crystal Fibers}

The predominant method for fabricating PCFs with a lattice of air holes is the stack-andpull procedure ${ }^{1}$. Rods and tubes of silica glass are stacked and pulled to a fiber in a drawing tower. The relative position of the rods and tubes in the stack determines the geometry of the lattice, while the inner and outer radius of the tubes and the shrinkage during the pulling of the fiber controls the period and size of the holes. The core of an index guiding PCF is defined simply by replacing a tube with a solid rod. This fabrication procedure allows the experienced manufacturer to produce highly advanced fiber structures including fibers with multiple cores. An alternative method for manufacturing PCFs in soft materials is the extrusion method ${ }^{2}$, where the fiber material is extruded through a mask, which defines the air holes.

\section{Optical properties of Photonic Crystal Fibers}

The optical properties of PCFs fabricated from pure silica are determined by the position, geometry and size of the air holes. A commonly accepted classification of PCFs divides the fibers into two main classes: 1) index-guiding PCFs and 2) Photonic Band Gap (PBG)

\footnotetext{
${ }^{1}$ J.C. Knight et al., "All-silica single-mode optical fiber with photonic crystal cladding", Opt. Lett., vol. 21, no. 19, pp. 1547-1549, 1996.

${ }^{2}$ K.M. Kiang et al., "Extruded singlemode non-silica glass holey optical fibers", Electron. Lett., vol. 38, no. 12 , pp. 546-547, 2002.
} 
guiding fibers ${ }^{3,4}$. In an index-guiding PCF the refractive index of the core is higher than the effective refractive index of the cladding and the fiber operates by the principle of total internal reflection. PBG fibers require a lattice of holes with a photonic band gap at the frequency, at which the fiber is intended to transmit light. Due to the different nature of the guiding mechanisms, the two classes of PCFs are treated in individual sections.

\section{Index-guiding PCF}

A common feature of all index-guiding PCFs is the solid silica core surrounded by a microstructured cladding. Due to the presence of the air holes the effective refractive index of the cladding is below that of the core and light is guided through the principle of total internal reflection. Three examples of index guiding PCFs are depicted in Fig. 1. The optical properties of index-guiding PCFs with a simple lattice of air holes, such as the Large Mode Area (LMA) and the Highly Nonlinear (HNL) fibers seen in Fig. 1a and Fig. $1 \mathrm{~b}$, are primarily determined by the geometry and pitch, $\Lambda$, of the lattice, and the air hole diameter, $d$. One of the novel properties of PCFs, compared to conventional fibers, is the phenomenon of endlessly single mode guidance ${ }^{5}$ observed in fibers with air holes arranged in a triangular pattern and $d / \Lambda \leq 0.406^{6,7}$. This behavior can be explained by considering the normalized frequency, $V$, used to estimate the number of guided modes in a step index fiber ${ }^{8}$

$$
V=\frac{2 \pi a}{\lambda}\left(n_{\text {core }}^{2}-n_{\text {clad }}^{2}\right)^{1 / 2}
$$

Here $\lambda$ is the wavelength, $a$ the core radius, $n_{\text {core }}$ the refractive index of the core and the effective refractive index of the cladding, $n_{\text {clad }}$, is calculated from the fundamental space filling mode of the infinite triangular air hole lattice. In conventional fibers, the frequency dependence of $n_{\text {clad }}$ is negligible and the number of guided modes grows with decreasing wavelength. In a PCF, the frequency dependence of $n_{\text {clad }}$ can be sufficiently strong to counteract the $1 / \lambda$ factor, thus keeping $V$ below the threshold for the first higher-order mode in a wide range of wavelengths.

One of the fields, where PCFs in particular has proven their superiority over conventional fibers is within the fabrication of highly non-linear fibers ${ }^{9}$. The presence of air holes in the cladding introduces a large index step at the core-cladding interface. This large index step provides a strong confinement of the guided mode, thereby, yielding high mode-field

\footnotetext{
${ }^{3}$ T.A. Birks et al., "Full 2-D photonic bandgaps in silica/air structures", Electron. Lett., vol. 31, no. 22, pp. 1941-1943, 1995.

${ }^{4}$ J.C. Knight et al., "Photonic band gap guidance in optical fibers", Science, vol. 282, no.5393, pp. 14761478, 1998.

${ }^{5}$ T.A. Birks et al., "Endlessly single-mode photonic crystal fiber", Opt. Lett., vol 22., no 13, pp. 961-963. 1997.

${ }^{6}$ B.T. Kuhlmey, "Modal cutoff in microstructured optical fibers", Opt. Lett., vol. 27, no. 19, pp. 1686$1688,2002$.

${ }^{7}$ N.A. Mortensen et at., "Modal cut-off and the V-parameter in photonic crystal fibers", submitted to Opt. Lett.

${ }^{8}$ A. Snyder and J. Lowe, "Optical waveguide theory", Chapman and Hall, 1983.

${ }^{9}$ N.G.R Broderick et al., "Nonlinearity in holey optical fibers: measurement and future opportunities", Opt. Lett., vol. 24, no. 20, pp. 1395-1397, 1999.
} 
intensity in the core. Furthermore, the extended freedom in the design of the optical properties of PCFs makes the fabrication of PCFs, with reduced dispersion in a wide wavelength range ${ }^{10,11}$ or an anomalous dispersion at visible wavelengths ${ }^{12}$; possible. Such fibers can exhibit non-linear effects, which are significantly stronger than what has been reported in conventional fibers, where the chromatic dispersion of the fiber limits the strength or the spectral bandwidth of the non-linear effects. A picture of a PCF optimized with respect to obtaining a high non-linearity is seen in Fig. 1b.

The holes in an index-guiding fiber does not necessary have to be positioned in a lattice. An example of this is the High Numerical Aperture (High NA) fiber seen in Fig. 1c, which only has a single ring of holes. The small width of the silica bridges connecting the core and the outer cladding reduce the effective refractive index of the PCF cladding to a level below 1.3, depending on the thickness of the bridges. The refractive index of the core equals that of pure silica (around 1.45), and this fiber geometry exhibits index steps between core and cladding superior to what can be obtained by doping core and cladding in conventional multimode fibers. Since the numerical aperture depends on this index step, an exceptionally high numerical aperture characterizes this kind of PCF. Fibers with an outer diameter of $125 \mu \mathrm{m}$ exhibiting a NA above 0.7 have been fabricated.

Selectively doping the core or regions of this with various materials such as Germanium or other refractive index changing elements influences the optical properties of the PCF. An excellent example of a PCF with modified properties is the highly non-linear dispersion-shifted fiber demonstrated by Hansen et $a l^{11}{ }^{11}$, who fabricated a triangular core PCF with an up-doped core center surrounded by three down-doped regions and three holes. This fiber shows fully controllable dispersion level and slope over a wide range of wavelengths. An additional advantage gained by doping the fiber is the resulting sensitivity towards intense ultraviolet exposure. This opens up for the fabrication of grating based PCF devices such as Bragg reflectors ${ }^{13}$ and Long Period Gratings used for instance as stress and temperature sensors ${ }^{14}$.

\section{Photonic Band Gap fibers}

The second class of photonic crystal fibers guide light by the photonic band gap effect $^{15,16}$. PBG guidance of optical signals in PCFs was first proposed in 1995 by Birks

10 A. Ferrando et al., "Designing the properties of dispersion-flattened photonic crystal fibers", Opt. Express, vol 9. no 13, pp.687-697, 2001.

${ }^{11}$ K.P. Hansen et al., "Fully dispersion controlled triangular-core nonlinear photonic crystal fiber", Optical Fiber Communication Conference OFC'03, Post Deadline paper (Atlanta, Georgia, March 2003).

12 J.K. Ranka et al., "Visible continuum generation in air-silica microstructure optical fibers with anomalous dispersion at 800nm", Opt. Lett. Vol. 25, no. 1, pp. 25-27, 2000.

${ }^{13}$ T. Erdogan "Fiber grating spectra", Jour. of Lightwave Tech., vol. 15, no. 8, pp. 1277-1295, 1997.

${ }^{14}$ V. Bhathi et al, "Optical fiber long-period grating sensors", Opt. Lett., vol. 21, no. 9, pp. 962-694, 1996.

${ }^{15}$ E.Yablonovitch, "Inhibited spontaneous emission in solid-state physics and electronics", Phys. Rev. Lett. vol. 58, no. 20, pp. 2059-62, 1987.

${ }^{16}$ S.John, "Strong localization of photons in certain disordered dielectric lattices", Phys. Rev. Lett., vol.58, no. 23, pp. 2486-89, 1987. 
et $a l^{3}$, who considered a PCF with the air holes arranged in a triangular lattice. In order to obtain PBG guidance in a PCF fiber, the air-hole lattice is designed to have at least one complete out-of-plane photonic band gap, in which propagation of light in the crystal plane is prohibited. Fig. 2 shows a modal index illustration of the photonic band diagram for a honeycomb PBG fiber with $d / \Lambda=0.8$. The out-of-plane wave vector component, hole size, lattice geometry and refractive index of the background material determine the width and the position of the individual band gaps. A fiber core is defined by introducing a defect that supports modes with frequencies within one of the photonic band gaps. The refractive index of the defect is below that of the surrounding cladding. This is one of the most interesting features of PBG fibers, since it opens up the unique possibility of guiding light in a hollow core.

Two fiber geometries in particular have been investigated in the work reported on PBG fibers. In 1998, Knight et al. ${ }^{4}$ presented the first experimental results on PBG guidance in air-silica fibers. They observed PBG guidance with strong band gaps in a PCF with the air holes arranged in a honeycomb lattice. Fibers with this lattice structure exhibit strong bands with relatively small air-filling fractions and are hence more easily fabricated than triangular PBG fibers, which require a large $d / A$ ratio in order to show strong bands. Fig. $3 a$ and Fig. $3 b$ shows pictures of fibers with the honeycomb and triangular lattice geometry, respectively. Triangular PBG fibers ${ }^{17,18}$ have the advantage over honeycomb fibers that they more easily can be designed to guide the optical field in a hollow core. Even though the honeycomb fiber seen in Fig. 3a operates by the PBG effect, the majority of the optical field is still guided in the silica surrounding the defect air hole. The fabrication of PBG fibers has matured to a level, where several companies ${ }^{19,20,21}$ are capable of manufacturing triangular PBG fibers, which guide light with the majority of the optical field confined in the hollow core.

\section{Bragg fibers}

A different type of band gap fiber is the so-called Bragg fiber, which guide light by Bragg reflection from a cylindrical symmetric Bragg grating in the cladding. Such fibers have been used for guidance of radiation from a high power $\mathrm{CO}_{2}$ laser at wavelengths deep into the mid-IR range ${ }^{22}$, where a conventional solid core silica fiber would show a substantial loss.

\footnotetext{
${ }^{17}$ R.F. Cregan et al., "Size-mode photonic band gap guidance of light in air", Science, vol. 285, no. 5433, pp.1537-1539, 1999.

18 T.P. Hansen et al., "Air-guidance-over $345 \mathrm{~m}$ of large-core photonic bandgap fiber", Optical Fiber Communication Conference OFC'03, Post Deadline paper (Atlanta, Georgia, March 2003).

${ }^{19}$ Crystal Fibre A/S: http://www.crystal-fibre.com

${ }^{20}$ BlazePhotonics: http://www.blazephotonics.com

${ }^{21}$ Corning: http://www.corning.com

${ }^{22}$ B. Temelkuran et al. "Wavelength-scalable hollow optical fibres with large photonic bandgaps for $\mathrm{CO}_{2}$ laser transmission", Nature, vol. 420, no 6916, pp. 650-653, 2002.
} 


\section{Photonic Crystal Fibers for sensor applications}

Especially two features of the photonic crystal fibers have encouraged their use within sensors. One is the possibility of obtaining long interaction lengths between light propagating in the fiber and very small volumes of gasses or liquids positioned in the air holes. The other is the freedom in the design of the optical properties of the fibers including the number of guiding cores. This section briefly describes some of the sensor applications of PCFs reported so far.

\section{Evanescent wave sensing.}

Modeling of the modes guided through an index-guiding PCF confirms that a significant part of the evanescent field can penetrate into the air holes of a carefully designed fiber ${ }^{23}$. The strength of the evanescent field and the long interaction length makes index-guiding PCFs interesting for evanescent wave sensing devices. Hoo et al. ${ }^{24}$ measured the absorption spectrum of acetylene by inserting one end of a 75-centimeter long PCF into a pressure chamber filled with $100 \%$ acetylene gas. The fiber was subsequently removed and the absorption spectrum was measured quickly in order to minimize out-diffusion of the gas. The fiber used in this experiment had a relatively weak penetration of the optical field into the air holes, but the long interaction length provided by the PCF efficiently compensated this. Jensen et $a l^{25}$ detected the presence of Cy5-labeled DNA in less than $1 \mu \mathrm{L}$ sample volume. A fiber section of 10 centimeters was filled with sample using capillary forces only and the transmission spectrum of the fiber was measured. When the sample contains the labeled DNA, the transmission spectrum shows dips at wavelengths corresponding to the absorption bands of the Cy5 molecule. Fig. 4 shows the absorption spectrum of the Cy5 label molecules derived from the measured transmission spectrum.

\section{Enhanced fluorescense biosensing}

A very elegant use of PCFs for sensor applications is the improved detection efficiency within two-photon fluorescence detection compared to conventional single mode fibers by using a double-clad fiber ${ }^{26}$. The light used to excite the chromophore is guided in the core defined by a defect in the triangular lattice of the inner cladding. The outer ring of air holes defines a core with a very high numerical aperture, which effectively collects the fluorescence emitted form the exited biomolecules.

\section{Bend sensing}

In the stack-and-pull method used in the production of PCFs a high-index core can be defined by replacing a tube with a solid rod at the appropriate lattice position. Fabrication of multi-core fibers is hence no more complicated than fabrication of single-core fibers.

\footnotetext{
${ }^{23}$ T. Monro et al., "Developing holey fibers for evanescent field devices", Electron. Lett., vol.35, no. 14, pp. 1188-1189, 1999.

${ }^{24}$ Y.L. Hoo et al., "Evanescent-wave gas sensing using microstructure fiber", Opt. Eng., vol 41, no 1, pp. 89, 2002.

${ }^{25}$ J.B. Jensen et al., " Photonic crystal fiber based evanescent-wave sensor for detection of biomolecules in aqueous solutions", to be presented at: Conference on Lasers and Electro Optics 2003, CLEO/QELS2003 Baltimore, USA.

${ }^{26}$ M.T. Myaing et al., "Enhanced two-photon biosensing with double-clad photonic crystal fibers", Opt. Lett., vol. 28, no. 14, pp. 1224-1226, 2003
} 
Blanchard et $a l^{27}$ demonstrated two-dimensional bend sensing using a PCF with three weakly interacting single-mode guiding cores. A deformation of the fiber introduces a phase shift between the beams propagating in the different cores. The bend angle is then determined by analyzing the fringes in the resulting far field interference pattern.

\section{Non-linear properties}

The freedom in the design of the optical properties and the strong confinement of the optical field within the core of index-guiding PCFs allows for strong non-linear effects as described above. One of the most intensively studied of these is the supercontinuum generation $^{12}$. A short intense pulse generates a wide spectrum of wavelengths, which can be used in optical coherence tomography ${ }^{28}$, spectroscopy ${ }^{29}$ and metrology ${ }^{30}$. Supercontinua covering several hundred nanometers as well as multi-Watt output have been demonstrated ${ }^{31}$.

\section{Endlessly single mode operation}

The endlessly single mode guidance seen in certain PCFs as described above is advantageous for spectroscopy applications, where light in a very broad range of wavelengths probes the sample. Another manifestation of the endlessly single mode guidance is the large mode areas that can be obtained, while still operating in single mode $^{8}$. The scalability of the PCF ensures that the number of modes is determined by the $d / \Lambda$ ratio rather than the core size. PCFs with large mode areas, such as the one illustrated in Fig. 1a, can hence deliver single-mode high power beams, while generating only insignificant nonlinear effects.

Hybrid photonic crystal fibers.

Another favorable property of PCFs is the possibility of fabricating hybrid components with materials like polymers or high index fluids in the air holes of the fiber ${ }^{32}$. Abramov et al $^{33}$ obtained a wide tuning range of a long-period grating by filling a polymer with a highly temperature dependent refractive index into the air holes of the fiber. The

${ }^{27}$ P.M. Blanchard et al., "Two-dimensional bend sensing with a single, multi-core optical fibre", Smart Materials and Structures, vol.9, no. 2, pp. 132-140, 2000.

${ }^{28}$ I Hartl at al., "Ultrahigh-resolution optical coherence tomography using continuum generation in an airsilica microstructure optical fiber", Opt. Lett., vol 26, nr. 9, pp. 608-610, 2001.

${ }^{29}$ V. Nagarajan et al., "A compact versatile femtosecond spectrometer", Rew. Sci. Instrum., vol. 73, no. 12, pp. 4145-4149, 2002.

${ }^{30}$ S.A. Diddams et al., "A compact femtosecond-laser-based optical clockwork", in Las. Freq. Stab., Stand., Meas., and Appl., Proc. of SPIE, vol. 4269, pp. 77-83, 2001.

${ }^{31}$ P.A. Champert et al., "Generation of multiwatt, broadband continua in holey fibers", Opt. Lett., vol. 27, no. 2, pp.122-124, 2002.

${ }^{32}$ C. Kerbage et al., "Microstructured optical fibers for integrated tunability of photonic devices", Opt. \& Phot. News, vol. 13, no 9, pp. 38-42, 2002.

${ }^{33}$ A.A. Abramov et al., "Widely tunable long-period fibre gratings", Electron. Lett., vol. 35, no. 1, pp. 8182, 1999. 
resonance wavelength, $\lambda_{\text {clad,i, }}$, of the coupling between the $L P_{01}$. mode and the $\mathrm{i}^{\text {th }}$ cladding modes changes according to

$$
\lambda_{\text {clad, }}(T)=\left[n_{\text {core }}-n_{\text {clad,i }}(T)\right] \Lambda
$$

Here $n_{\text {core }}$ and $n_{\text {clad,i }}$ are the effective refractive indices of the core mode and the $i$ 'th cladding mode, respectively, $T$ is the temperature, and $A$ is the periodicity of the grating. Due to the temperature dependence of the refractive index of the polymer, the effective refractive index of the cladding modes and hence the resonance wavelength changes significantly with temperature. The sensitivity towards changes in the temperature was $74 \mathrm{~nm} / 100^{\circ} \mathrm{C}$, which is superior to the changes obtained in the fiber without the polymer in the holes $\left(3 \mathrm{~nm} / 100^{\circ} \mathrm{C}\right)$ and LPG temperature sensors based on conventional solid fibers $^{14}$. The strong sensitivity of the resonance wavelength towards changes in the temperature can evidently be utilized to realize a highly sensitive fiber based temperature sensor.

\section{Atom guides}

It is possible to guide atoms through a PCF by generating a magnetic field from driving a current through wires placed in adjacent air holes ${ }^{34}$. Single atom guidance will open up for a range of highly sensitive sensors for measuring physical quantities, which influence the motion of the atom. Examples of potential applications are gravitational, rotational and magnetic field sensors.

\section{PBG fiber based sensors.}

The majority of the sensor applications of PCFs reported in the literature is based on index-guiding fibers. A significant advantage of PBG fibers for sensor applications is the possibility of guiding light in hollow cores filled with a gas or aqueous solutions of molecules. In a carefully designed PBG, the majority of the mode field is guided in the sample volume, thus providing a strong interaction between light and molecules. A PBG fiber based device for absorption measurements can evidently provide a strong interaction over several tens of centimeters, while using only a few micro liters of sample volume. The index-guiding PCF also has a significant interaction between the guided light and molecules present in the air holes, but is it only the evanescent field, which penetrates into the holes. With the improved guiding properties and quality of the commercially available PBG fibers, it is expected that the number of PBG fiber based sensors will increase in the near future.

\section{Conclusion}

In conclusion, we have described some of the improved or novel optical properties provided by photonic crystal fibers and summarized their applications within sensors. The distribution and size of the air holes determine the optical properties, thus allowing

\footnotetext{
${ }^{34}$ M. Key et al. "Propagation of cold atoms along a miniature magnetic guide", Phys. Rev. Lett., vol. 84, no. 7, pp. 1371-1373, 2000.
} 
the production of fibers with a wide range of attractive properties such as endlessly single-mode guidance, large mode area, high numerical aperture, controllable dispersion, photonic band gap guidance as well as multiple-core fibers. Several applications of these properties within sensors are mentioned. A characteristic feature of photonic crystal fibers, which makes these particular interesting for sensor applications is the close proximity of samples positioned in the air holes and light guided through the fiber. Sensors based on evanescent-wave sensing in index-guiding PCFs has already been realized and it is expected that with the improved skills of the manufacturers, sensors based on photonic band gap fibers will emerge soon.

\section{Acknowledgements}

The author has benefited from useful discussions with T.P. Hansen, K.P. Hansen, and M.D. Nielsen.

\section{Figures}

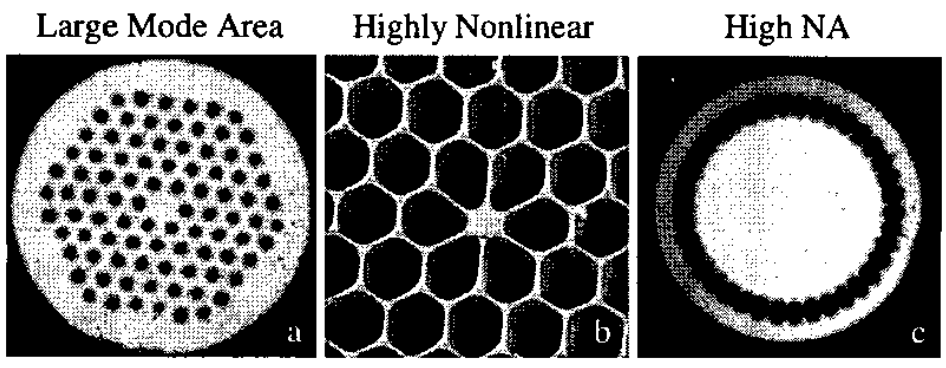

Fig. 1: Three different index-guiding photonic crystal fibers. Pictures courtesy of Crystal Fibre A/S 


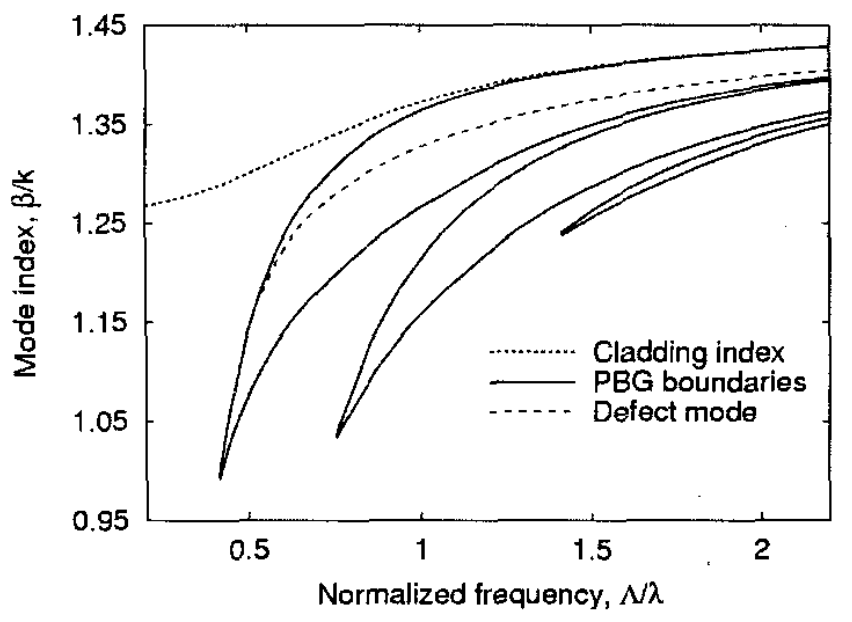

Fig. 2: Mode-index illustration of the photonic band gaps exhibited by a honeycomb fiber with $d=0.8 \Lambda$. The defect, which is defined by an additional hole with $d_{\mathrm{co}}=0.3 \Lambda$, supports one mode within the first band gap.
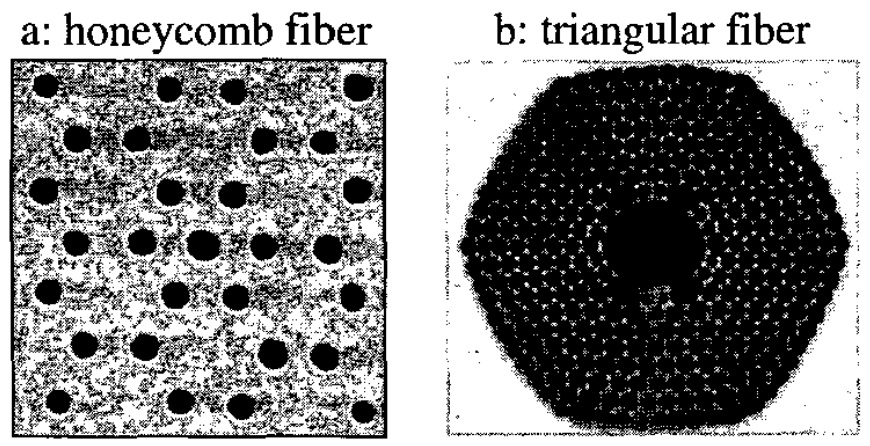

Fig. 3: Photonic band gap fibers with the air holes positioned in a): a honeycomb lattice and b): a triangular lattice. Pictures courtesy of Crystal Fibre A/S 


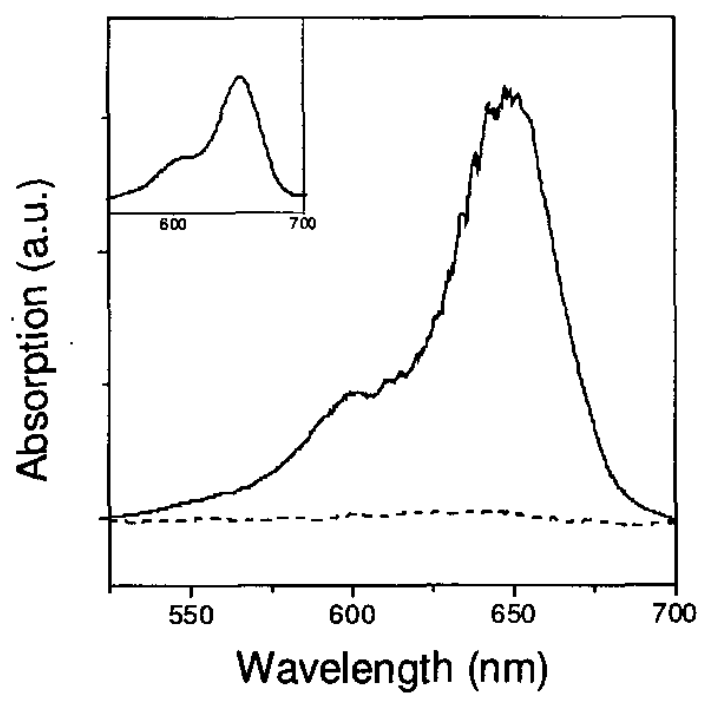

Fig. 4: Absorption spectrum of the Cy5 label molecule derived from the transmission spectrum of a PCF filled with a $0.8 \mathrm{~mL}$ aqueous solution of Cy5-labeled DNA (solid line) and a reference measurement on pure water (dashed line). The insert shows the Cy5 absorption spectrum measured on a conventional spectrometer 